\title{
REGULARITY OF THE EXTREMAL SOLUTION IN A MEMS MODEL WITH ADVECTION*
}

\author{
CRAIG COWAN $^{\dagger}$ AND NASSIF GHOUSSOUB ${ }^{\ddagger}$
}

Abstract. We consider the regularity of the extremal solution of the nonlinear eigenvalue problem

$$
(S)_{\lambda} \quad\left\{\begin{aligned}
-\Delta u+c(x) \cdot \nabla u & = & \frac{\lambda}{(1-u)^{2}} & \text { in } \Omega, \\
u & = & 0 & \text { on } \partial \Omega,
\end{aligned}\right.
$$

where $\Omega$ is a smooth bounded domain in $\mathbb{R}^{N}$ and $c(x)$ is a smooth bounded vector field on $\bar{\Omega}$. We show that, just like in the advection-free model $(c \equiv 0)$, all semi-stable solutions are smooth if (and only if) the dimension $N \leq 7$. The novelty here comes from the lack of a suitable variational characterization for the semi-stability assumption. We overcome this difficulty by using a general version of Hardy's inequality. The same method applies for the Gelfand problem (i.e., exponential nonlinearity).

Key words. Extremal solution, regularity.

AMS subject classifications. 35J60, 35B32, 35D10, 35J20

1. Introduction. The following equation has often been used to model a simple Micro-Electro-Mechanical System (MEMS) device:

$$
(P)_{\lambda} \quad\left\{\begin{aligned}
-\Delta u & =\frac{\lambda}{(1-u)^{2}} & & \text { in } \Omega, \\
u & =0 & & \text { on } \partial \Omega,
\end{aligned}\right.
$$

where $\Omega$ is a smooth bounded domain in $\mathbb{R}^{N}, \lambda>0$ is proportional to the applied voltage and $0<u(x)<1$ denotes the deflection of the membrane. This model has been extensively studied, see [9], [10] in regards to the model and [6], [5], [7] for mathematical aspects of $(P)_{\lambda}$. It is well known (see above references) that there exists some positive finite critical parameter $\lambda^{*}$ such that for all $0<\lambda<\lambda^{*}$, the equation $(P)_{\lambda}$ has a smooth minimal stable (see below) solution $u_{\lambda}$, while for $\lambda>\lambda^{*}$ there are no weak solutions of $(P)_{\lambda}$ (see [6] for a precise definition of weak solution). Standard elliptic regularity theory yields that a solution $u$ of $(P)_{\lambda}$ is smooth if and only if $\sup _{\Omega} u<1$. One can also show that $\lambda \mapsto u_{\lambda}(x)$ is increasing and hence one can define the extremal solution

$$
u^{*}(x):=\lim _{\lambda \nearrow \lambda^{*}} u_{\lambda}(x),
$$

which can be shown to be a weak solution of $(P)_{\lambda^{*}}$.

Recall that a smooth solution $u$ of $(P)_{\lambda}$ is said to be minimal if any other solution $v$ of $(P)_{\lambda}$ satisfies $u \leq v$ a.e. in $\Omega$. Such solutions are then semi-stable meaning that the principal eigenvalue of the linearized operator

$$
L_{u, \lambda}:=-\Delta-\frac{2 \lambda}{(1-u)^{3}}
$$

\footnotetext{
* Received August 10, 2008; accepted for publication October 21, 2008.

$\dagger$ Department of Mathematics, University of British Columbia, Vancouver, B.C. Canada V6T 1Z2 (ctcowan@math.ubc.ca). This work is part of the author's PhD dissertation in preparation under the supervision of N. Ghoussoub.

${ }^{\ddagger}$ Department of Mathematics, University of British Columbia, Vancouver, B.C. Canada V6T $1 Z 2$ (nassif@math.ubc.ca). Research partially supported by the Natural Science and Engineering Research Council of Canada.
} 
in $H_{0}^{1}(\Omega)$ is nonnegative. This property can be expressed variationally by the inequality

$$
2 \lambda \int_{\Omega} \frac{\psi^{2}}{(1-u)^{3}} \leq \int_{\Omega}|\nabla \psi|^{2}, \quad \forall \psi \in H_{0}^{1}(\Omega)
$$

which can be viewed as the nonnegativeness of the second variation of the energy functional associated with $(P)_{\lambda}$ at $u$.

Now a question of interest is whether $u^{*}$ is a smooth solution of $(P)_{\lambda^{*}}$. It is shown in [6] that this is indeed the case provided $N \leq 7$. This result is optimal in the sense that $u^{*}$ is singular in dimension $N \geq 8$ with $\Omega$ taken to be the unit ball.

Our main interest here will be in the regularity of the extremal solution associated with

$$
(S)_{\lambda} \quad\left\{\begin{aligned}
-\Delta u+c(x) \cdot \nabla u & = & \frac{\lambda}{(1-u)^{2}} & \text { in } \Omega \\
u & = & 0 & \text { on } \partial \Omega
\end{aligned}\right.
$$

where $c \in C^{\infty}\left(\bar{\Omega}, \mathbb{R}^{N}\right)$ and where again $\Omega$ is a smooth bounded domain in $\mathbb{R}^{N}$. Modifying the proofs used in analyzing $(P)_{\lambda}$ one can again show the existence of a positive finite critical parameter $\lambda^{*}$ such that for $0<\lambda<\lambda^{*}$ there exists a smooth minimal solution $u_{\lambda}$ of $(S)_{\lambda}$, while there are no smooth solutions of $(S)_{\lambda}$ for $\lambda>\lambda^{*}$. Moreover, the minimal solutions are also semi-stable in the sense that the principal eigenvalue of the corresponding linearized operator

$$
L_{u, \lambda, c}:=-\Delta+c(x) \cdot \nabla-\frac{2 \lambda}{\left(1-u_{\lambda}\right)^{3}}
$$

in $H_{0}^{1}(\Omega)$ is non-negative. See [3] where these results are proved for general $C^{1}$ convex nonlinearities which are superlinear at $\infty$. Our main result concerns the regularity of the extremal solution of $(S)_{\lambda}$.

Theorem 1. If $1 \leq N \leq 7$, then the extremal solution $u^{*}$ of $(S)_{\lambda^{*}}$ is smooth.

REMARK 1. A crucial (in fact the main) ingredient in proving the regularity of $u^{*}$ in $(P)_{\lambda}$, is the energy inequality (1) which is used in conjunction with the equation $(P)_{\lambda}$, to obtain uniform (in $\left.\lambda\right) L^{p}$-estimates on $\left(1-u_{\lambda}\right)^{-2}$ whenever $u_{\lambda}$ is the minimal solution (See [6]). However, the semi-stability of $u_{\lambda}$ in the case of $(S)_{\lambda}$, does not translate into an energy inequality which allows the use of arbitrary test functions. Overcoming this will be the major hurdle in proving Theorem 1.

We point out, however, that if $c(x)=\nabla \gamma$ for some smooth function $\gamma$ on $\bar{\Omega}$, then the semi-stability condition on the minimal solution $u_{\lambda}$ of $(S)_{\lambda}$ translates into

$$
2 \lambda \int_{\Omega} \frac{e^{-\gamma} \psi^{2}}{\left(1-u_{\lambda}\right)^{3}} \leq \int_{\Omega} e^{-\gamma}|\nabla \psi|^{2}, \quad \forall \psi \in H_{0}^{1}(\Omega)
$$

Then, with slight modifications, one can use the standard approach for $(P)_{\lambda}$ to obtain the analogous result for $(S)_{\lambda}$ stated in Theorem 1 .

The novel case is therefore when $c$ is a divergence free vector field. Actually, we shall use the following version of the Hodge decomposition, in order to deal with general vector fields $c$. 
Lemma 1. Any vector field $c \in C^{\infty}\left(\bar{\Omega}, \mathbb{R}^{N}\right)$ can be decomposed as $c(x)=-\nabla \gamma+$ $a(x)$ where $\gamma$ is a smooth scalar function and $a(x)$ is a smooth bounded vector field such that $\operatorname{div}\left(e^{\gamma} a\right)=0$.

Proof. By the Krein-Rutman theory, the linear eigenvalue problem

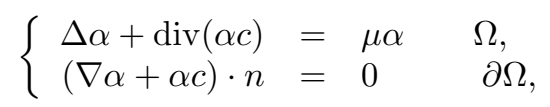

where $n$ is the unit outer normal on $\partial \Omega$, has a positive solution $\alpha$ in $\Omega$ when $\mu$ is the principal eigenvalue. Integrating the equation over $\Omega$, one sees that $\mu=0$. The positivity of $\alpha$ on the boundary follows from the boundary condition and the maximum principle. In other words, we have that $\Delta \alpha+\operatorname{div}(\alpha c)=0$ on $\Omega$, and $\alpha>0$ on $\bar{\Omega}$.

Now define $\gamma:=\log (\alpha)$ and $a:=c+\nabla \gamma$. An easy computation shows that $\operatorname{div}\left(e^{\gamma} a\right)=0$.

Throughout the rest of this note $c, a, \gamma$ will be defined as above.

2. A general Hardy inequality and non-selfadjoint eigenvalue problems. Consider the linear eigenvalue problem

$$
\left\{\begin{aligned}
-\Delta \phi+c \cdot \nabla \phi-\rho \phi & =K \phi & & \Omega \\
\phi & = & & 0
\end{aligned}\right.
$$

where $c$ is a smooth bounded vector field on $\Omega, \rho \in C^{\infty}(\bar{\Omega})$ and $K$ is a scalar. We assume that $(\phi, K)$ is the principal eigenpair for (4) and that $\phi>0$ in $\Omega$, and $K \geq 0$. Note that elliptic regularity theory shows that $\phi$ is then smooth.

We shall now use a general Hardy inequality to make up for the lack of a variational characterization for the pair $(\phi, K)$. The following result is taken from [4], which we duplicate here for the convenience of the reader. For a complete discussion on general Hardy inequalities including best constants, attainability and improvements of, see [4]. We should point out that this approach to Hardy inequalities is not new, but it is generally restricted to specific functions $E$ which yield known versions of Hardy inequalities; see [1] and reference within.

Lemma 2. Let $A(x)$ denote a uniformly positive definite $N \times N$ matrix with smooth coefficients defined on $\Omega$. Suppose $E$ is a smooth positive function on $\Omega$ and fix a constant $\beta$ with $1 \leq \beta \leq 2$. Then, for all $\psi \in H_{0}^{1}(\Omega)$ we have

$$
\int_{\Omega}|\nabla \psi|_{A}^{2} \geq \frac{\beta(2-\beta)}{4} \int_{\Omega} \frac{|\nabla E|_{A}^{2}}{E^{2}} \psi^{2}+\frac{\beta}{2} \int_{\Omega} \frac{-\operatorname{div}(A \nabla E)}{E} \psi^{2},
$$

where $\int_{\Omega}|\nabla \psi|_{A}^{2}=\int_{\Omega} A(x) \nabla \psi \cdot \nabla \psi$.

Proof. For simplicity we prove the case where $A(x)$ is given by the identity matrix. For the general case, we refer to [4]. Let $E_{0}$ denote a smooth positive function defined in $\Omega$ and let $\psi \in C_{c}^{\infty}(\Omega)$. Set $v:=\frac{\psi}{\sqrt{E_{0}}}$. Then

$$
|\nabla \psi|^{2}=E_{0}|\nabla v|^{2}+\frac{\left|\nabla E_{0}\right|^{2}}{4 E_{0}^{2}} \psi^{2}+v \nabla v \cdot \nabla E_{0} .
$$

Integrating the last term by parts gives

$$
\int_{\Omega} v \nabla v \cdot \nabla E_{0}=\frac{1}{2} \int_{\Omega} \frac{-\Delta E_{0}}{E_{0}} \psi^{2}
$$


and so integrating (6) gives

$$
\int_{\Omega}|\nabla \psi|^{2} \geq \frac{1}{4} \int_{\Omega} \frac{\left|\nabla E_{0}\right|^{2}}{E_{0}^{2}} \psi^{2}+\frac{1}{2} \int_{\Omega} \frac{-\Delta E_{0}}{E_{0}} \psi^{2},
$$

where we dropped a nonnegative term. So we have the desired result for $\beta=1$. When $\beta \neq 1$ one puts $E_{0}:=E^{\beta}$ into (7) and collects like terms to obtain the desired result.

口

We now use the above lemma to obtain an energy inequality valid for the principal eigenpair of (4).

THEOREM 2. Suppose that the principal eigenpair $(\phi, K)$ of (4) are such that $\phi>0$ and $K \geq 0$. Then, for $1 \leq \beta \leq 2$ we have for all $\psi \in H_{0}^{1}(\Omega)$,

$$
\int_{\Omega} e^{\gamma}|\nabla \psi|^{2} \geq \frac{\beta(2-\beta)}{4} \int_{\Omega} \frac{e^{\gamma}|\nabla \phi|^{2}}{\phi^{2}} \psi^{2}+\frac{\beta}{2} \int_{\Omega} e^{\gamma} \rho(x) \psi^{2}-\frac{\beta}{2} \int_{\Omega} \frac{e^{\gamma} a \cdot \nabla \phi}{\phi} \psi^{2} .
$$

Proof. Note that (4) can be rewritten as

$$
-\operatorname{div}\left(e^{\gamma} \nabla \phi\right)+e^{\gamma} a \cdot \nabla \phi=e^{\gamma}(\rho+K) \phi \quad \text { in } \Omega,
$$

where as mentioned above we are using the decomposition $c=-\nabla \gamma+a$. We now set $E:=\phi$ and $A(x)=e^{\gamma} I$ (where $I$ is the identity matrix) and use (5) along with the above equation to obtain the desired result. Note that we have dropped the nonnegative term involving $K$.

3. Proof of theorem 1. For $0<\lambda<\lambda^{*}$, we denote by $u_{\lambda}$ the smooth minimal semi-stable solution of $(S)_{\lambda}$. Let $(\phi, K)$ denote the principal eigenpair associated with the linearization of $(S)_{\lambda}$ at $u_{\lambda}$. Then $0<\phi$ in $\Omega, 0 \leq K$ and $(\phi, K)$ satisfy

$$
\left\{\begin{array}{rlrl}
-\Delta \phi+c \cdot \nabla \phi & =\left(\frac{2 \lambda}{\left(1-u_{\lambda}\right)^{3}}+K\right) \phi & \Omega \\
\phi & =0 & & \partial \Omega
\end{array}\right.
$$

Again, elliptic regularity theory shows that $\phi$ is smooth. Consider $c=-\nabla \gamma+a$ to be the decomposition of $c$ described in Lemma 1. We now obtain the main estimate.

Theorem 3. For $0<\lambda<\lambda^{*}, 1<\beta<2$ and $0<t<\beta+\sqrt{\beta^{2}+\beta}$, we have the following estimate:

$$
\lambda\left(\beta-\frac{t^{2}}{2 t+1}\right) \int_{\Omega} \frac{e^{\gamma}}{\left(1-u_{\lambda}\right)^{2 t+3}} \leq 2 \beta \lambda \int_{\Omega} \frac{e^{\gamma}}{\left(1-u_{\lambda}\right)^{t+3}}+\frac{\beta\|a\|_{L^{\infty}}^{2}}{4(2-\beta)} \int_{\Omega} \frac{e^{\gamma}}{\left(1-u_{\lambda}\right)^{2 t}} .
$$

Proof. Fix $0<\beta<2$, let $0<t$ and $u$ denote the minimal solution associated with $(S)_{\lambda}$. We shall use Theorem 2 with $\rho(x)=\frac{2 \lambda}{\left(1-u_{\lambda}\right)^{3}}$. Put $\psi:=\frac{1}{(1-u)^{t}}-1$ into (8) to obtain

$$
\begin{aligned}
t^{2} \int_{\Omega} \frac{e^{\gamma}|\nabla u|^{2}}{(1-u)^{2 t+2} \geq} & \beta \lambda \int_{\Omega} \frac{e^{\gamma}}{(1-u)^{3}}\left(\frac{1}{(1-u)^{t}}-1\right)^{2} \\
& +\frac{\beta}{2} \int_{\Omega} e^{\gamma}\left(\frac{(2-\beta)}{2} \frac{|\nabla \phi|^{2}}{\phi^{2}}-\frac{a \cdot \nabla \phi}{\phi}\right) \psi^{2}
\end{aligned}
$$

Now note that $(S)_{\lambda}$ can be rewritten as

$$
-\operatorname{div}\left(e^{\gamma} \nabla u\right)+e^{\gamma} a \cdot \nabla u=\frac{\lambda e^{\gamma}}{(1-u)^{2}} \quad \text { in } \Omega,
$$


and test this on $\bar{\phi}:=\frac{1}{(1-u)^{2 t+1}}-1$ to obtain

$$
(2 t+1) \int_{\Omega} \frac{e^{\gamma}|\nabla u|^{2}}{(1-u)^{2 t+2}}+H=\lambda \int_{\Omega} \frac{e^{\gamma}}{(1-u)^{2}}\left(\frac{1}{(1-u)^{2 t+1}}-1\right)
$$

where

$$
H:=\int_{\Omega} e^{\gamma} a \cdot \nabla u\left(\frac{1}{(1-u)^{2 t+1}}-1\right) .
$$

One easily sees that $H=0$ after considering the fact $H$ can be rewritten in the form $\int_{\Omega}\left(e^{\gamma} a\right) \cdot \nabla G(u)$ for an appropriately chosen function $G$ with $G(0)=0$. Combining the above two inequalities and dropping some positive terms gives

$$
\begin{aligned}
\lambda\left(\beta-\frac{t^{2}}{2 t+1}\right) \int_{\Omega} \frac{e^{\gamma}}{(1-u)^{2 t+3}} \leq & 2 \beta \lambda \int_{\Omega} \frac{e^{\gamma}}{(1-u)^{3+t}} \\
& +\frac{\beta}{2} \int_{\Omega} e^{\gamma} \Lambda(x)\left(\frac{1}{(1-u)^{t}}-1\right)^{2}
\end{aligned}
$$

where

$$
\Lambda(x):=\frac{a \cdot \nabla \phi}{\phi}-\frac{(2-\beta)}{2} \frac{|\nabla \phi|^{2}}{\phi^{2}} .
$$

Simple calculus shows that

$$
\sup _{\Omega} \Lambda(x) \leq \frac{\|a\|_{L^{\infty}}^{2}}{2(2-\beta)}
$$

which, after substituting into the above inequality, completes the proof of the main estimate. $\square$

Note now that the restriction $t<\beta+\sqrt{\beta^{2}+\beta}$ is needed to ensure that the coefficient $\beta-\frac{t^{2}}{2 t+1}$ is positive. It follows then that $\frac{1}{\left(1-u_{\lambda}\right)^{2}}$ is uniformly bounded (in $\lambda)$ in $L^{p}(\Omega)$ for all $p<p_{0}:=\frac{7}{2}+\sqrt{6} \approx 5.94 \ldots$ and after passing to limits we have the same result for the extremal solution $u^{*}$.

To conclude the proof of Theorem 1, it suffices to note the following result.

Lemma 3. Suppose $3 \leq N \leq 7$ and the extremal solution $u^{*}$ satisfies $\frac{1}{\left(1-u^{*}\right)^{2}} \in$ $L^{\frac{3 N}{4}}(\Omega)$, then $u^{*}$ is smooth.

Proof. First note that by elliptic regularity one has $u^{*} \in W^{2, \frac{3 N}{4}}(\Omega)$ and after applying the Sobolev embedding theorem one has $u^{*} \in C^{0, \frac{2}{3}}(\bar{\Omega})$. Now suppose $\|u\|_{L^{\infty}}=1$ so that there is some $x_{0} \in \Omega$ such that $u\left(x_{0}\right)=1$. Then

$$
\frac{1}{1-u(x)} \geq \frac{C}{\left|x-x_{0}\right|^{\frac{2}{3}}}
$$

and hence

$$
\infty>\int_{\Omega} \frac{1}{\left(\left(1-u^{*}\right)^{2}\right)^{\frac{3 N}{4}}} \geq C \int_{\Omega} \frac{1}{|x|^{N}}=\infty
$$

which is a contradiction. It follows that $\frac{1}{\left(1-u^{*}\right)^{2}} \in L^{\infty}(\Omega)$, and $u^{*}$ is therefore smooth. 
Proof of Theorem 1. Using this lemma and the above $L^{p}$-bound on $\frac{1}{\left(1-u^{*}\right)^{2}}$, one sees that $u^{*}$ is smooth for $3 \leq N \leq 7$. To show the result in dimensions $N=1,2$, one needs a slight variation of the above argument. We omit the details, and the interested reader can consult [6] for the proof when $c(x)=0$.

The same approach works on the following Gelfand problem with advection:

$$
(T)_{\lambda} \quad\left\{\begin{array}{rlr}
-\Delta u+a(x) \cdot \nabla u & =\lambda e^{u} & \text { in } \Omega, \\
u & =0 & \text { on } \partial \Omega,
\end{array}\right.
$$

where $\Omega$ is a bounded domain in $\mathbb{R}^{N}$ with smooth boundary and where $a$ is a smooth bounded vector field. For simplicity we assume that $a$ is divergence free. Then one has the following result:

Theorem 4. Suppose $N \leq 9$. Then the extremal solution associated with $(T)_{\lambda}$ is smooth.

Proof. Let $1<t<\beta<2$ and $0<\lambda<\lambda^{*}$. Setting $\psi:=e^{t u_{\lambda}}-1$ and letting $\bar{\phi}$ be a suitable multiple of $e^{2 t u_{\lambda}}-1$, follow the proof of Theorem 1 to obtain the inequality

$$
\begin{aligned}
\lambda(\beta-t) \int_{\Omega} e^{(2 t+1) u_{\lambda}} \leq & \beta \int_{\Omega}\left(\frac{a \cdot \nabla \phi}{\phi}-\frac{(\beta-2)}{2} \frac{|\nabla \phi|^{2}}{\phi^{2}}\right)\left(e^{t u_{\lambda}}-1\right)^{2} \\
& +2 \beta \lambda \int_{\Omega} e^{(t+1) u_{\lambda}},
\end{aligned}
$$

where $0<\phi \in H_{0}^{1} \cap C^{\infty}$ is the positive principal eigenfunction associated with the linearized problem at $u_{\lambda}$. As before one can easily obtain the estimate that for all $1<t<2$ there is some $C_{t}<\infty$ such that

$$
\int_{\Omega} e^{(2 t+1) u_{\lambda}} \leq C_{t}
$$

uniformly in $\lambda$. Using elliptic regularity theory along with the Sobolev imbedding theorem one sees that $u_{\lambda}$ is uniformly bounded in $L^{\infty}(\Omega)$ provided $N \leq 9$ which gives the desired result after passing to limits.

\section{REFERENCES}

[1] Adimurthi And Anusha Sekar, Role of the fundamental solution in Hardy-Sobolev-type inequalities, Proceedings of the Royal Society of Edinburgh, 136A (2006), pp. 1111-1130.

[2] X. Cabré and A. Capella, Regularity of radial minimizers and extremal solutions of semilinear elliptic equations, J. Funct. Anal., 238:2 (2006), pp. 709-733.

[3] H. Berestycki, A. Kiselev, A. Novikov and L. Ryzhik, The explosion problem in a flow, preprint (2008).

[4] C. Cowan, Optimal Hardy inequalities for general elliptic operators with improvements, to appear in Commun. Pure Appl. Anal.

[5] P. Esposito, N. Ghoussoub And Y. Guo, Compactness along the branch of semi-stable and unstable solutions for an elliptic problem with a singular nonlinearity, Comm. Pure Appl. Math., 60 (2007), pp. 1731-1768.

[6] N. Ghoussoub And Y. Guo, On the partial differential equations of electro MEMS devices: stationary case, SIAM J. Math. Anal., 38 (2007), pp. 1423-1449.

[7] Y. GuO, Z. PAN AND M.J. WARD, Touchdown and pull-in voltage behavior of a mems device with varying dielectric properties, SIAM J. Appl. Math., 66 (2005), pp. 309-338.

[8] G. Nedev, Regularity of the extremal solution of semilinear elliptic equations, C. R. Math. Acad. Sci. Paris, 330 (2000), pp. 997-1002.

[9] J.A. Pelesko, Mathematical modeling of electrostatic mems with tailored dielectric properties, SIAM J. Appl. Math., 62 (2002), pp. 888-908.

[10] J.A. Pelesko And A.A. Berstein, Modeling MEMS and NEMS, Chapman Hall and CRC Press, 2002. 